\title{
Comments on Statistical Errors for May $\quad$ commentary Issue 2012
}

\section{Yong Gyu Park}

Department of Biostatistics, The Catholic University of Korea College of Medicine, Seoul, Korea

In this section, we would like to explain the properties of the relative risk (RR) and odds ratio (OR) which appeared in the article titled, "Misinterpreting odds ratio in the articles published in the Korean Journal of Family Medicine," published in March 2012 by Kim et al., ${ }^{1)}$ and the relationship between both measures in prospective and retrospective studies.

\section{Odds Ratio and Relative Risk in Prospective/ Retrospective Studies}

We assume that the following hypothetical census data are obtained in a certain community to investigate the effect of smoking on lung cancer. We also assume that the total population is 100,000 people and the smoking rate is $40 \%$.

\begin{tabular}{lccc}
\hline & Lung cancer & Normal & Total \\
\hline Smoker & 4,000 & 36,000 & 40,000 \\
Non-smoker & 3,000 & 57,000 & 60,000 \\
Total & 7,000 & 93,000 & 100,000 \\
\hline
\end{tabular}

In this case, RR is calculated as $\frac{4,000 / 40,000}{3,000 / 60,000}=\frac{0.1}{0.05}=2$, which indicates that the prevalence of lung cancer for the smoking group is twice that of the non-smoking group. On the other hand, RR, which can be obtained by the equation $\frac{4,000 / 36,000}{3,000 / 57,000}=\frac{0.1 / 0.9}{0.05 / 0.95} \approx$ 2.1, has a similar value to the relative risk. Because RR and OR have nearly the same values when the frequencies of a specific event are very small and the size of the total population is very large, both measures are interpreted as the same in the field of epidemiology, which mostly deals with data having the above characteristics.
Now consider that the study is conducted prospectively with 2,000 smokers and 2,000 non-smokers who are randomly selected from each population, respectively. The results are summarized as follows:

\begin{tabular}{lccc}
\hline & Lung cancer & Normal & Total \\
\hline Smoker & 200 & 1,800 & 2,000 \\
Non-smoker & 100 & 1,900 & 2,000 \\
Total & 300 & 3,700 & 4,000 \\
\hline
\end{tabular}

In the above table, the frequencies of lung cancer for each group, $10 \%$ and 5\%, respectively, are obtained from census data, and are quite acceptable,considering the fact that both groups are selected from each population by random sampling. From the above results,

$$
\begin{aligned}
& \mathrm{RR}=\frac{200 / 2,000}{100 / 2,000}=\frac{0.1}{0.05}=2, \\
& \mathrm{OR}=\frac{200 / 1,800}{100 / 1,900}=\frac{0.1 / 0.9}{0.05 / 0.95} \approx 2.1,
\end{aligned}
$$

the same values as in the census data.

If we perform the study retrospectively, with 2,000 lung cancer patients and 2,000 normal controls who are randomly selected from patient and normal populations, respectively, then the results are summarized as follows:

\begin{tabular}{lrcc}
\hline & Lung cancer & Normal & Total \\
\hline Smoker & 1,143 & 774 & 1,917 \\
Non-smoker & 857 & 1,226 & 2,083 \\
Total & 2,000 & 2,000 & 4,000 \\
\hline
\end{tabular}

In the above table, the proportions of smoker for each 
group, $57 \%(\approx 4,000 / 7,000)$ and $39 \%(\approx 36,000 / 93,000)$, respectively, are obtained from census data, and again, are quite acceptable,considering the fact that both groups are selected from each population by random sampling. From the above results,

$$
\begin{aligned}
& \mathrm{RR}=\frac{1,143 / 1,917}{857 / 2,083}=\frac{0.596}{0.411} \approx 1.45 \\
& \mathrm{OR}=\frac{1,143 / 774}{857 / 1,225}=\frac{0.596 / 0.404}{0.411 / 0.589} \approx 2.1 .
\end{aligned}
$$

This shows that the value of RR, 1.45 from a retrospective study, could be very different from those from census data and prospective studies. However, OR had the same values, 2.1, irrespective of the study designs.

Another unavoidable drawback of a retrospective study is that the proportions of lung cancer for smokers and non-smokers, $60 \%(\approx 1,143 / 1,917)$ and $41 \%(\approx 857 / 2,083)$, are unrealistically large.

To solve such deficiencies of a retrospective study, we could obtain RR after adjusting the prevalence of smoking in the population. This is referred to as Bayes theorem we will skip further explanation here because there are so many textbooks concerning this subject.

Kim et al. ${ }^{1)}$ pointed out the misinterpretation of OR in the articles published in the Korean Journal of Family Medicine as $\mathrm{RR}$ and proposed the formula of Zhang and $\mathrm{Yu},{ }^{2}$ )

$$
\text { Estimated } R R=\frac{O R}{\left(1-P_{0}\right)+\left(P_{0} \times O R\right)}
$$

which could be used to calculate RR from OR, where $\mathrm{P}_{0}$ denotes the proportion of occurrence of an event for the control group.

We can obtain RR by substituting the results of above prospective study into this formula as follows.

Estimated $R R=\frac{2.11}{(1-0.05)+(0.05 \times 2.11)}=\frac{2.11}{1.055} \approx 2$.

However, this formula can only be used for ORs which are obtained from prospective studies. For ORs obtained from retrospective studies, the relation between estimated $R R$ and $O R$ is not expressed in the above formula, and the prevalence rate of smoking in the population should be additionally adjusted.

\section{CONFLICT OF INTEREST}

No potential conflict of interest relevant to this article was reported.

\section{REFERENCES}

1. Kim JH, Kim MY, Kim SY, Hwang IH, Kang EJ. Misinterpreting odds ratio in the articles published in Korean Journal of Family Medicine. Korean J Fam Med 2012;33:8993.

2. Zhang J, Yu KF. What's the relative risk? A method of correcting the odds ratio in cohort studies of common outcomes. JAMA 1998;280:1690-1. 